\section{Avaliação Psicológica: Definição de aspectos psicométricos segundo alunos de graduação em psicologia}

\author{
Bruno Bonfá-Araujo
}

Eliana Santos de Farias

Janaina Chnaider Miranda

\section{RESUMO}

A compreensão da avaliação psicológica é uma importante ferramenta a qualquer profissional de Psicologia. 0 objetivo desta pesquisa foi analisar o conhecimento em avaliação psicológica de alunos do curso de Psicologia. Participaram 150 estudantes universitários, $79,3 \%$ se declararam do sexo feminino, sendo de duas universidades particulares do estado de São Paulo, de diversos semestres. Foram utilizados o Conhecimento de Avaliação Psicológica (CAP), composto por cinco perguntas abertas e o Questionário de Competências para Avaliação Psicológica (QCAP). Os resultados indicaram que conceitos essenciais ainda são mal compreendidos, como aspectos psicométricos e a função do SATEPSI, constatando uma falta de conhecimento teórico-prático no que diz respeito ao processo de avaliação psicológica. Deste modo, é necessário pensar criticamente a grade de disciplinas dos cursos, estratégias e recursos de ensino que possam melhorar a formação e atuação em território nacional.

Palavras-chave: diretrizes de ensino; formação do psicólogo; testagem psicológica.

\section{ABSTRACT}

\section{Psychological Assessment: Definition of psychometric aspects according to undergraduate students in psychology}

The knowledge of psychological assessment is an important tool for any Psychology professional. The objective of this research was to analyze the knowledge of the psychological assessment in undergraduate students of Psychology. Participated 150 undergraduates, $79.3 \%$ declared themselves female, being from two private universities in the state of São Paulo, from several semesters. We used the Knowledge of Psychological Assessment (CAP), composed of five open questions and the Competence Questionnaire for Psychological Assessment (QCAP). The results indicated that essential concepts are still poorly understood, such as psychometric aspects and the SATEPSI function, evidencing a lack of theoretical-practical knowledge regarding the psychological assessment process. Thus, it is necessary to think critically about the discipline of courses, strategies and teaching resources that can improve the training and performance in national territory.

Keyword: teaching guidelines; psychologists' education; psychological testing.

\section{Sobre os Autores}

B. B.

https://orcid.org/0000-0003-

0702-9992

Universidade São Francisco,

Campinas - São Paulo

brunobonffa@outlook.com

E. S. F.

ORCID: https://orcid.org/00000001-7715-7012

Centro Universitário São Camilo -

CUSC, São Paulo

elianass@gmail.com

J. C. M.

https://orcid.org/0000-0001-

5567-6698

PUC - Campinas, Campinas -

São Paulo

jana_chnaider@hotmail.com

\section{Direitos Autorais}

Este é um artigo aberto e pode ser reproduzido livremente, distribuído, transmitido ou modificado, por qualquer pessoa desde que usado sem fins comerciais. O trabalho é disponibilizado sob a licença Creative Commons CC-BY-NC 


\section{W NTERAC̄̃OEM PSICOLOGIA \\ Bruno Bonfá-Araujo, Eliana Santos de Farias e Janaina Chnaider Miranda}

A avaliação psicológica (AP) é um processo amplo e complexo que exige do psicólogo um grande conhecimento teórico e técnico (Borsa, 2016). 0 processo de AP constitui o uso de técnicas, estratégias e métodos psicológicos, sendo a única atividade privativa do psicólogo garantida na lei que regulamenta a profissão (ver artigo 13, §1 da lei 4.119/1962). A prática da AP é fiscalizada e passível de punição em caso de violações e uso impróprio, a partir da criação do Sistema Conselhos - ConseIho Federal de Psicologia (CFP) e Conselhos Regionais de Psicologia (CRP) (Presidência da República, Casa Civil, Subchefia para Assuntos Jurídicos, 1962, 1971).

Mesmo assim, durante muitos anos a formação em AP no Brasil foi considerada insatisfatória (Ambiel et al., 2019). Principalmente a partir da década de 1990, docentes e psicólogos atuantes em diversos contextos se mobilizaram com o objetivo de melhorar a preparação dos alunos de graduação em Psicologia no que se refere à formação em avaliação psicológica (Alchieri \& Cruz, 2003; Noronha et al., 2014).

Visando a contribuir com o desenvolvimento técnico e científico dos psicólogos que trabalham com AP, assim como assegurar o respeito da prática com seres humanos, o Conselho Federal de Psicologia determinou, por meio da Resolução 009/2018 (que revoga a 002/2003, 006/2004 e 005/2012), que é responsabilidade dos cursos de Psicologia oferecer um plano de ensino que garanta o ensino apropriado do manejo e compreensão dos testes psicológicos (CFP, 2018).

Essa resolução, com o objetivo de reafirmar a qualidade técnico científica de instrumentos psicológicos, reestabeleceu que os testes psicológicos são instrumentos de uso exclusivo do psicólogo, estabelecendo diretrizes que norteiam a atuação do psicólogo no que se refere à avaliação psicológica em diversos contextos, como trânsito (e.g., Santos et al., 2018), escolar (e.g., Gomes et al., 2018; Marques et al., 2018; Nakano et al., 2010), clínico (e.g., Campos, 2017) e esporte (e.g., Garcia \& Borsa, 2016), dentre outros.

No Brasil, embora haja leis, resoluções e documentos que determinam padrões para construção e uso de testes psicológicos (e.g., American Educational Research Association [AERA], American Psychological Association [APA] \& National Council on Measurment in Education [NCME], 2014; CFP, 2018), ainda não há diretrizes que determinem a necessidade de especialização do psicólogo para o manuseio de qualquer que seja o instrumento e sua complexidade, como ocorre com os profissionais estadunidenses, por exemplo (Noronha et al., 2014). Nesse contexto, cabe aos psicólogos o bom senso, o comprometimento profissional e uma ética deontológica baseada em buscar conhecimento e domínio dos testes utilizados em sua prática e práxis profissional, a qual, em geral, ocorre informalmente por meio de grupos de estudos ou cursos complementares.

Ainda que a AP no Brasil tenha tido avanços desde a década de 1990 (Alchieri \& Cruz, 2003), a formação dos psicólogos, de uma forma geral, é deficiente. Muitos psicólogos atuam nesta área sem uma formação técnica e teórica necessária (Borsa, 2016). As disciplinas que abrangem a avaliação psicológica são, em sua maioria, ministradas por docentes especialistas, mestres ou doutores formados fora da especificidade da AP (Ambiel et al., 2018). Sugerindo a possibilidade de haver falta de preparo dos docentes, por sua vez relacionada ao pouco investimento que o psicólogo faz em seu aprimoramento profissional depois da graduação, afetando, consequentemente o nível de qualidade de ensino, o que alimenta um tipo de ciclo vicioso (profissionais mal formados, ensino ruim, prática ruim) (Ambiel et al., 2017; Mendes et al., 2013).

No que se refere à pesquisa em avaliação psicológica, a vinculação de linhas de pesquisa a importantes instituições de ensino, tem contribuído para o grande aumento de publicações na área (Borsa, 2016). De modo que, mais de $80 \%$ dos docentes de AP estão envolvidos em atividades de atualização profissional (Bardagi et al., 2015). Em contrapartida, de acordo com Mendes et al. (2013), a falta de uma diretriz específica sobre o plano de ensino em AP, assim como o pouco interesse dos docentes em se atualizar, são os responsáveis pela defasagem no desenvolvimento de competências e habilidades dos graduandos em Psicologia.

0 conhecimento da definição sobre critérios psicométricos é tão essencial como o domínio das atualizações desses conceitos. Reforçando a necessidade da continuidade dos estudos dos profissionais em avaliação psicológica após a graduação, uma vez que é comum a estes profissionais concluírem a graduação com um conhecimento superficial para a sua prática e práxis profissional (Mendes et al., 2013). De modo que, mesmo durante o período de formação, os graduandos tendem a avaliar conceitos da área como importantes, porém consideram ao longo da formação que pouco dominam a temática, fato esse que pode se expandir para a prática profissional (Noronha et al., 2009).

Uma falha no processo de formação e avaliação de clientes, mesmo que não proposital, pode contribuir para 
uma rotulação do sujeito, bem como para o processo de exclusão (Moura, 2017). Nesse sentido, o Conselho Federal de Psicologia prevê como falha ética do profissional a não consideração dos critérios estabelecidos pela Resolução 009/2018, além de avaliações pautadas em resultados aos quais o profissional não possui domínio (CFP, 2018).

Pensando nessas questões que influenciam uma formação mais especializada para o fazer em AP, um exemplo pode ser considerado. A Universidade Federal do Rio Grande do Sul estabeleceu um espaço para a prática de seus graduandos em Psicologia, denominado de Centro de Avaliação Psicológica (CAP), sendo o principal objetivo deste a possibilidade de aprendizado teórico-técnico-prático aos futuros profissionais (Borsa et al., 2013).

Outras instituições lançam mão de outras estratégias como oficinas, atendimentos de casos clínicos e monitorias em avaliação psicológica que permitem ao formando vivenciar, melhor e fora da sala de aula, as necessidades, limites e dificuldades do fazer profissional (e.g. Fernandes et al., 2015; Janasi et al., 2017; Rabelo et al., 2016; Souza \& Barboza, 2014). Outros autores propõem que as instituições de ensino superior (IES), por meio de seus professores de AP, possam permitir espaços e situações em que os alunos possam experienciar, desenvolver criticidade, criatividade e outras competências no fazer de avaliação psicológica. Estas estratégias podem contribuir para desmistificar a prática e ajudar a desenvolver um pensamento mais crítico e contextualizado, visto que, às vezes, esse aluno terá que escolher outros recursos para além dos testes com parecer favorável pelo SATEPSI. Este fato se dá devido a não termos instrumentos que atendam aos critérios mínimos para uso estabelecidos pelo CFP que possibilitem investigar todos os construtos no âmbito psicológico (CFP, 2018; Moura, 2017; Noronha \& Reppold, 2010).

Desta maneira deve-se, portanto, considerar a necessidade de atualização de formação e ensino da avaliação psicológica no Brasil. São diversas as adversidades enfrentadas no processo de ensino deste tópico, partindo da relação aluno-professor até o caráter tecnicista com enfoque apenas no psicodiagnóstico (Bardagi et al., 2015; Freires et al., 2017; Gouveia, 2018). Desta forma, é imprescindível uma melhor sistematização para o ensino do tópico de maneira nacional.

A partir do exposto e inspirado pelo estudo de Mendes et al. (2013), objetivou-se investigar o conhecimento de graduandos de Psicologia sobre os critérios psicométricos, bem como identificar o nível de conhecimento sobre avaliação psicológica dos alunos em concordância com os semestres no qual estão inseridos. A priori, hipotetizou-se que, conforme encontrado no estudo de Mendes et al. (2013), os estudantes de Psicologia têm uma visão distorcida sobre os conceitos da avaliação psicológica.

\section{MÉTODO}

\section{PARTICIPANTES}

Participaram do estudo 150 estudantes universitários do estado de São Paulo na região Sudeste do país, todos alunos do curso de Psicologia de duas universidades privadas. Do total de participantes $38 \%$ estavam matriculados no terceiro semestre, $16 \%$ no segundo, $14,7 \%$ no sexto, $12,7 \%$ no quinto, $12 \%$ no décimo, $2 \%$, respectivamente, no sétimo, oitavo e nono semestre e $0,7 \%$ no quarto semestre. A faixa etária variou entre 18 e 55 anos ( $M$ = 24,99; $D P=8,45) ; 79,3 \%$ se declarou do sexo feminino, $e$ $20,7 \%$ do sexo masculino.

\section{INSTRUMENTOS}

Questionário de identificação: elaborado com o objetivo de identificar características dos participantes, como idade, sexo e semestre.

Roteiro com itens sobre o Conhecimento de Avaliação Psicológica (CAP; Mendes et al., 2013): Composto por seis questões que objetivam avaliar o conhecimento de estudantes de Psicologia sobre avaliação psicológica, a saber: 1 - Como você define a avaliação psicológica?; 2 Quais os métodos que o psicólogo pode usar durante um processo de avaliação psicológica?; 3 - Você sabe qual a função do SATEPSI, criado pelo CFP?; 4 - Quais os requisitos mínimos para um teste estar autorizado para o uso do psicólogo?; 5 - 0 que você sabe sobre validade de um teste?; 6 - 0 que você sabe sobre precisão de um teste?

O instrumento CAP foi corrigido a partir de um tipo de rubrica sobre respostas esperadas e aceitáveis (pré-estabelecidas), elaboradas a partir do estudo de Mendes et al. (2013), sendo pontuado no seguinte formato: 0 - "Não sabe ou não respondeu", 1 - "Não apresentou nenhum dos conceitos esperados", 2 - "Apresentou um dos conceitos esperados", 3 - "Apresentou dois ou todos os conceitos 


\section{- 4 * INTERACÃO EM 4. PSICOLOGIA \\ Bruno Bonfá-Araujo, Eliana Santos de Farias e Janaina Chnaider Miranda}

esperados". Foram consideradas as seguintes respostas para os outros itens: 1- $A$ avaliação psicológica é definida pelo Conselho Federal de Psicologia (2018) como um processo técnico e científico de coleta de dados, estudos e interpretação de informações a respeito dos fenômenos psicológicos; 2 - O psicólogo pode fazer uso de estratégias psicológicas assim como métodos, técnicas e instrumentos; 3 - O SATEPSI é uma norma de certificação de instrumentos de avaliação psicológica que avalia e qualifica os instrumentos em apto ou inapto para uso profissional, a partir da verificação objetiva de um conjunto de requisitos técnicos mínimos; 4 - Fundamentação teórica, estudos sobre propriedades psicométricas, principalmente sobre validade e precisão, bem como informações sobre seu sistema interpretativo; 5 - Um instrumento é válido quando mede o que supostamente se propõe medir e 6 - Um instrumento é preciso quando ele mede sem erros, de maneira que os escores obtidos pela mesmo pessoa em ocasiões diferentes ou em diferentes conjuntos de itens equivalentes têm que ser coerentes.

Questionário de Competência para Avaliação Psicológica (QCAP; Noronha et al., 2007): Composto por 20 afirmações, que objetivam identificar a "Importância" e o "Domínio" que os estudantes têm em relação à avaliação psicológica, os itens são respondidos em formato tipo $\mathrm{Li}$ kert de três pontos para cada fator ("nada" a "muito importante" e "nenhum" até "muito domínio"). Destaca-se que todos os itens são respondidos para ambos os fatores, sendo que o fator Importância retrata o nível de relevância atribuído ao conteúdo dos itens, assim como o fator Domínio representa a competência dos respondentes no que diz respeito às afirmações. $A$ análise fatorial indicou seis dimensões, três para o fator Domínio (Aplicação da avaliação psicológica; Construção de testes psicológicos; Utilização de um teste psicológico na avaliação psicológica) e três para o fator Importância (Aplicação da avaliação psicológica; Correção e intepretação de testes psicológicos; Construção de testes psicológicos). Os índices de precisão do instrumento indicaram um alfa de Cronbach que variaram entre 0,87 para a escala total de Importância e 0,93 para a escala total de Domínio (Rueda et al., 2009). Alguns exemplos de itens são, 1 - "Aplicar os princípios éticos na avaliação psicológica"; 10 - "Interpretar tabelas dos manuais de testes" e 19 - "Conhecer e fazer uso das condições adequadas para a testagem e aplicação dos instrumentos".

\section{PROCEDIMENTOS}

O projeto foi aprovado pelo Comitê de Ética em Pesquisa (CAAE: 89822418.9.0000.5497), assim como as autoras dos instrumentos foram consultadas para utilização destes. Após aprovação foi construído um formulário na plataforma Google Forms, sendo este divulgado no Facebook, em grupos específicos de Psicologia e avaliação psicológica. 0 período de coleta de dados ocorreu nos meses de setembro e outubro de 2018. Para responder ao questionário de identificação e aos dois instrumentos, o respondente deveria inicialmente concordar com as informações apresentadas no Termo de Consentimento Livre e Esclarecido (TCLE), não sendo necessário identificar-se pelo nome. Estima-se que o tempo necessário para responder aos instrumentos era de 30 minutos.

Todos os estudantes realizaram o Conhecimento de Avaliação Psicológica (CAP), contudo apenas 36 realizaram o Questionário de Competências para Avaliação Psicológica (QCAP). Como não havia a condição obrigatória de responder ao QCAP no formulário online, inclusive por esta condição não ir ao encontro do objetivo principal deste estudo, alguns estudantes optaram, de modo individual e espontâneo, por não responder ao instrumento.

\section{ANÁLISE DE DADOS}

Os dados foram analisados no software Statistical Package for Social Sciences (SPSS), versão 23. Para a categorização da amostra, foram utilizadas estatísticas descritivas, o cálculo de média e desvio padrão foi realizado para ambos instrumentos.

\section{RESULTADOS}

A partir do objetivo de compreender o conhecimento de avaliação psicológica de estudantes de Psicologia, foram analisadas as médias dos alunos por meio de dois instrumentos. A Tabela 1 apresenta os resultados de seis questões abertas, englobando desde itens mais simples como a definição da avaliação psicológica, até itens mais complexos como o conceito de validade e precisão. 


\section{W' INTERACÃO EM PSICOLOGIA \\ Bruno Bonfá-Araujo, Eliana Santos de Farias e Janaina Chnaider Miranda}

Tabela 1: Média das questões do Conhecimento de Avaliação Psicológica (CAP)

\begin{tabular}{lccc}
\hline & $n$ & $M$ & $D P$ \\
\hline 1. Definição da AP & 150 & 1,85 & 0,79 \\
2. Métodos utilizados & 150 & 1,93 & 1,25 \\
em AP & 150 & 0,89 & 1,10 \\
3. SATEPSI & 150 & 1,39 & 0,98 \\
$\begin{array}{l}\text { 4. Requisitos para o uso } \\
\text { de testes }\end{array}$ & 150 & 0,87 & 1,01 \\
5. Validade & 150 & 0,94 & 0,87 \\
6. Precisão & & & \\
\hline
\end{tabular}

Considerando os dados encontrados, apenas os itens 1 e 2 ficaram acima da média, sendo estes a definição da avaliação psicológica e quais estratégias o psicólogo pode utilizar durante o processo de avaliação. Os outros quatro itens tiveram resultados abaixo da média, sendo o item 5 (a definição do conceito de validade) o de pior resultado, outro item que aparece com uma média abaixo do esperado é o item 3 , sobre a função do SATEPSI. A Tabela 2 apresenta as médias dos participantes no QCAP, indicadas a partir das duas dimensões principais do instrumento (Importância e Domínio).

No que diz respeito à Importância, os resultados que apresentaram maiores médias foram a aplicação dos princípios éticos, a interpretação precisa dos instrumentos psicológicos e as condições e comunicação do processo de testagem. Por outro lado, itens como fundamentação teórica e processo de construção dos instrumentos foram considerados menos importantes. A dimensão Domínio apresentou uma maior média em itens como a aplicação dos princípios éticos e a capacidade de compreensão dos estudos de validade e precisão, já as menores médias foram apresentadas em itens como o processo de construção de instrumentos e experiência supervisionada em avaliação.

\section{DISCUSSÃO}

Ao considerar os objetivos desta pesquisa, bem como os dados coletados e analisados, sobressaiu-se preocupantemente que esses alunos, distribuídos em diversos momentos do curso de graduação em Psicologia, inclusive último semestre, souberam explicar apenas os concei- tos básicos sobre avaliação: definição e método. Assim como no estudo de Mendes et al. (2013), esses resultados são alarmantes porque os alunos, uma vez formados, usarão a avaliação psicológica aonde quer que atuem.

Esse estudo ressalva o que foi apontado por Moura (2017), quando fala da necessidade de desmistificar a avaliação psicológica para os graduandos. Muitos verbalizaram escolher o curso de Psicologia por não ter afinidade com disciplinas que requerem cálculo e/ou habilidade numérica. Assim, quanto aos critérios básicos para que um teste possa ter parecer favorável por meio do SATEP$\mathrm{SI}$, como evidências de validade e precisão, bem como para buscar estes critérios nos instrumentos e entendê-los nos manuais de testes psicológicos, os alunos parecem alienar-se das definições científicas, sem demonstrar competências para escolher, de modo consciente e responsável, o instrumento que melhor atende sua necessidade profissional.

Por outro lado, ao se considerarem os resultados com o QCAP (Noronha et al., 2007), embora respondido por uma minoria dos participantes, as implicações parecem menos incoerentes, visto que as pontuações menores foram nos itens que tratam da construção de instrumentos. Espera-se que os graduandos em Psicologia sejam formados para conhecer as possibilidades e recursos para fazerem uma AP de qualidade, considerando todo o instrumental à disposição. No geral, não são formados para construir testes psicológicos. Este tipo de conhecimento é mais utilizado pelos profissionais que seguem seus estudos no stricto sensu, construindo uma carreira acadêmica-científica (Bandeira, 2018). Como aqueles com menor média em relação ao domínio foram itens de experiência supervisionada, reitera-se, assim como na literatura desse escrito, a necessidade de estratégias de ensino que possibilitem experiências reais como a monitoria e estágios supervisionados em avaliação psicológica que contemplem a etapa de testagem igualmente (Ambiel et al., 2017).

No mais, intenta-se para o fato mais amplo: a necessidade de melhoria no ensino de AP durante a graduação em Psicologia nas instituições de ensino superior (IES) brasileiras. Não basta que os docentes nas disciplinas de avaliação psicológica estejam atualizados, como apontado no estudo de Bardagi et al. (2015). Faz-se impreterível pensar criticamente a grade de disciplinas dos cursos, conteúdos, bibliografia, estratégias e recursos de ensino que alcancem os formandos a fim de melhorar a formação e atuação em território nacional. Faz-se necessário no ensino de Psicologia, perpassando inclusive pelos docentes de quaisquer outras disciplinas, que se compreenda que a avaliação psicológica é um processo amplo, que pode ou 
Tabela 2.: Média das questões do Questionário de Competência para Avaliação Psicológica (QCAP)

\begin{tabular}{|c|c|c|c|c|c|}
\hline \multirow{2}{*}{ Itens } & \multirow{2}{*}{$n$} & \multicolumn{2}{|c|}{ Importância } & \multicolumn{2}{|c|}{ Domínio } \\
\hline & & $M$ & $D P$ & $M$ & $D P$ \\
\hline $\begin{array}{l}\text { 1. Aplicação dos princí- } \\
\text { pios éticos }\end{array}$ & 36 & 3,00 & 0,00 & 2,11 & 0,82 \\
\hline $\begin{array}{l}\text { 2. Fundamentação } \\
\text { teórica }\end{array}$ & 36 & 2,81 & 0,47 & 1,67 & 0,63 \\
\hline 3. Psicopatologia & 36 & 2,92 & 0,28 & 1,61 & 0,64 \\
\hline $\begin{array}{l}\text { 4. Construtos psicoló- } \\
\text { gicos }\end{array}$ & 36 & 2,89 & 0,32 & 1,64 & 0,68 \\
\hline $\begin{array}{l}\text { 5. Gama de testes } \\
\text { psicológicos }\end{array}$ & 36 & 2,83 & 0,38 & 1,64 & 0,68 \\
\hline 6. Psicometria & 36 & 2,83 & 0,38 & 1,64 & 0,72 \\
\hline 7. Parecer psicológico & 36 & 2,92 & 0,28 & 1,72 & 0,74 \\
\hline $\begin{array}{l}\text { 8. Construção do ins- } \\
\text { trumento }\end{array}$ & 36 & 2,81 & 0,40 & 1,61 & 0,69 \\
\hline 9. Validade e precisão & 36 & 2,89 & 0,32 & 1,92 & 0,77 \\
\hline $\begin{array}{l}\text { 10. Interpretação de } \\
\text { manuais }\end{array}$ & 36 & 2,92 & 0,37 & 1,78 & 0,83 \\
\hline 11. Leitura de manuais & 36 & 2,89 & 0,32 & 1,86 & 0,76 \\
\hline $\begin{array}{l}\text { 12. Seleção de instru- } \\
\text { mentos }\end{array}$ & 36 & 2,89 & 0,40 & 1,78 & 0,80 \\
\hline 13. Estatística & 36 & 2,89 & 0,32 & 1,72 & 0,74 \\
\hline $\begin{array}{l}\text { 14. Usar e interpretar } \\
\text { instrumentos }\end{array}$ & 36 & 2,92 & 0,37 & 1,69 & 0,82 \\
\hline 15. Questões do cliente & 36 & 2,89 & 0,40 & 1,69 & 0,75 \\
\hline $\begin{array}{l}\text { 16. Interpretação } \\
\text { precisa }\end{array}$ & 36 & 2,97 & 0,17 & 1,89 & 0,85 \\
\hline $\begin{array}{l}\text { 17. Comunicação de } \\
\text { resultados }\end{array}$ & 36 & 2,94 & 0,33 & 1,89 & 0,89 \\
\hline 18. Aspectos legais & 36 & 2,89 & 0,40 & 1,92 & 0,87 \\
\hline $\begin{array}{l}\text { 19. Condições para } \\
\text { testagem }\end{array}$ & 36 & 2,94 & 0,33 & 1,83 & 0,81 \\
\hline $\begin{array}{l}\text { 20. Experiência super- } \\
\text { visionada }\end{array}$ & 36 & 2,92 & 0,37 & 1,56 & 0,81 \\
\hline Escala total & & 57,95 & 6,59 & 35,17 & 15,32 \\
\hline
\end{tabular}




\section{H'INTERACÃO EM PSICOLOGIA \\ Bruno Bonfá-Araujo, Eliana Santos de Farias e Janaina Chnaider Miranda}

não fazer uso de testes psicológicos. Deste modo, todos os profissionais ao realizarem um diagnóstico, avaliação, pesquisa, ou qualquer outro termo empregado nesse sentido, deveriam considerar aspectos científicos e éticos, amparados por leis federais e documentos reguladores do nosso conselho de classe, independente do uso de testes como um dos recursos para fazer AP e campo de trabalho.

Algumas limitações devem ser conceituadas para este estudo, a primeira delas diz respeito à quantidade e localização geográfica dos estudantes analisados. Estudos futuros devem buscar ampliar o número amostral, assim como coletar dados de estudantes de todas as regiões do Brasil para uma melhor compreensão do panorama nacional. A segunda limitação retrata o processo de correção subjetivo do instrumento Conhecimento de Avaliação Psicológica (CAP). Apesar de terem sido estabelecidos conteúdos para as respostas (rubricas), avaliadores diferentes podem atribuir pontuações diferentes. Sugere-se que o instrumento seja ampliado, de modo que possam ser indicadas respostas consideradas certas ou erradas, sem vieses de julgamento de valor.

\section{DECLARAÇÃO DE CONFLITOS DE INTERESSE}

Os autores declaram que não há conflitos de interesse no manuscrito submetido.

\section{REFERÊNCIAS}

Alchieri, J. C., \& Cruz, R. M. (2003). Avaliação psicológica: Conceito, métodos e instru- mentos. Casa do Psicólogo.

Ambiel, R. A. M., Baptista, M. N., Bardagi, M. P., \& Santos, A. A. A. (2018). Ensino de avaliação psicológica: Dificuldades relatadas por uma amostra de docentes brasileiros. Estudos e Pesquisas em Psicologia, 18(2), 515-530. doi: 10.12957/epp.2018.38810

Ambiel, R. A. M., Barros, L. O., Batista, H. H. V. (2017). Competências na Avaliação Psicológica de graduandos em psicologia: Análise do ensino e experiência em estágios. Psicologia Ensino \& Formação, 8(2), 3-13. https://doi.org/10.21826/2179-5800201782313

Ambiel, R. A., Zuanazzi, A. C., Sette, C. P., Costa, A. R. L., \& Cunha, F. A. (2019). Análise de ementas de disciplinas de Avaliação Psicológica: Novos tempos, velhas questões. Avaliação Psicológica, 18(1), 21-30. https://doi. org/10.15689/ap.2019.1801.15229.03
American Educational Research Association [AERA], American Psychological Association [APA], \& National Council on Measurment in Education [NCME] (2014). Standards for educational and psychological testing. American Educational Research Association.

Bandeira, D. R. (2018). A controvérsia do uso dos testes psicológicos por psicólogos e não psicólogos. Psicologia: Ciência e Profissão, 38, 159-166. https://doi. org/10.1590/1982-3703000208860

Bardagi, M. P., Teixeira, M. A. P., Segabinazi, J. D., Schelini, P. W., \& Nascimento, E. (2015). Ensino da Avaliação Psicológica no Brasil: levantamento com docentes de diferentes regiões. Avaliação Psicológica, 14(2), 253260. https://doi.org/10.15689/ap.2015.1402.10

Borsa, J. C., Oliveira, S. E. S., Yates, D. B., \& Bandeira, D. R. (2013). Centro de Avaliação Psicológica - CAP: Uma clínica-escola especializada em avaliação e diagnóstico psicológico. Psicologia Clínica, 25(1), 101-114. https://doi.org/10.1590/S0103-56652013000100007

Borsa, J. C. (2016). Considerações sobre a formação e a prática em Avaliação Psicológica No Brasil. Temas em Psicologia, 24(1), 131-143. https://doi.org/10.9788/ TP2016.1-09

Campos, R. C. (2017). Do processo de avaliação da personalidade em contextos clínicos ao diagnóstico psicodinâmico: Contributos para uma avaliação psicológica psicodinâmica. Revista Iberoamericana de Diagnóstico y Evaluación e Avaliação Psicológica, 2(44), 44-56. https://doi.org/10.21865/RIDEP44.2.04

Conselho Federal de Psicologia. (2018). Resolução CFP n. 009/2018: Estabelece diretrizes para a realização da Avaliação Psicológica no exercício profissional da psicóloga e do psicólogo, regulamente o Sistema de Avaliação de Testes Psicológicos - SATEPSI e revoga as Resoluções n. 002/2003, n. 006/2004 e n.005/2012 e Notas Técnicas n. 01/2017 e 02/2017. http://satepsi.cfp.org.br/docs/Resolu\%C3\%A7\%C3\%A3o-CFP-n\%C2\%BA-09-2018-com-anexo.pdf

Fernandes, M. A., Zerbinati, J. P., Cantares, T. S., \& Germano, G. S. (2015). Monitoria no ensino das paixões: Acolhimento ao aluno no primeiro contato com a psicopatologia. Analytica, 4(6), 138-150. http://pepsic. bvsalud.org/pdf/analytica/v4n6/07.pdf

Freires, L. A., Silva Filho, J. H., Monteiro, R. P., Loureto, G. D. L., \& Gouveia, V. V. (2017). Ensino de avaliação psicológica no norte brasileiro: Analisando as ementas das disciplinas. Avaliação Psicológica, 16(2), 205-214. https://doi.org/10.15689/AP.2017.1602.11 


\section{W' INTERACÃO EM

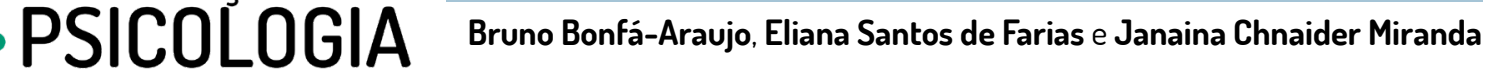

Garcia, R. P., \& Borsa, J. C. (2016). A prática da avaliação psicológica em contextos esportivos. Temas em Psicologia, 24(4), 1549-1560. https://doi.org/10.9788/ TP2016.4-20

Gomes, L. C., Silva, P. A., Farias, E. S., \& Anacleto, G. M. C. (2018). Avaliação do potencial criativo em crianças do ensino fundamental nos anos iniciais. Revista Científica UMC, edição especial PIBIC, 3(3). http://seer.umc. br/index.php/revistaumc/article/view/445/337

Gouveia, V. V. (2018). Formação em avaliação psicológica: Situação, desafios e diretrizes. Psicologia: Ciência e Profissão, 38(no.spe), 74-86. https://doi. org/10.1590/1982-3703000208641

Janasi, G. C., Araújo, B. B., \& Farias, E. S. (2017, 27-30 de Setembro). Monitoria universitária como estratégia de formação acadêmica em psicologia [Apresentação de trabalho]. Em M. P. R. Souza (Presidente), XIII Congresso nacional de psicologia escolar e educacional: Pela democratização da educação (CONPE), Salvador, BA. http://www.conpe2017.ufba.br/

Marques, N. D. S., Anacleto, G. M. C., \& Farias, E. S. (2018). Ansiedade e estatística: análise de produção científica. Revista Científica UMC, edição especial PIBIC, 3(3), 1-3.

Mendes, L. S., Nakano, T. C., Silva, I. B., \& Sampaio, M. H. L. (2013). Conceitos de avaliação psicológica: Conhecimentos de estudantes e profissionais. Psicologia: Ciência e Profissão, 33(2), 428-445. https://doi. org/10.1590/S1414-98932013000200013

Moura, D. P. F. (2017). O ensino de avaliação psicológica e as tendências pedagógicas: Possibilidades para um planejamento crítico. Revista Brasileira de Ensino Superior, 3(3), 42-53. https://doi.org/10.18256/24473944.2017.v3i3.2047

Nakano, T. C., Santos, E., Zavariz, S. F. Wechsler, S. M., \& Martins, E., (2010). Estilos de pensar e criar em universitários das áreas de humanas e sociais aplicadas: Diferenças por gênero e curso. Psicologia: Teoria e Prática, 12(3), 120-134. http://pepsic.bvsalud.org/pdf/ ptp/v12n3/v12n3a10.pdf

Noronha, A. P. P., Barros, M. V. C., Nunes, M. F. O., \& Santos, A. A. A. (2014). Avaliação psicológica: Importância e domínio de atividades segundo docentes. Estudos e Pesquisas em Psicologia, 14(2), 524-538. http:// pepsic.bvsalud.org/scielo.php?script=sci_arttext\&pi$d=S 1808-42812014000200008 \&$ Ing $=p t \& t \operatorname{lng}=p t$
Noronha, A. P. P., Nunes, M. F. O., Ambiel, R. A. M., \& Barros, M. V. C. (2007). Questionário de Competências para Avaliação Psicológica (QCAP). Universidade São Francisco.

Noronha, A. P. P., \& Reppold, C. T. (2010). Considerações sobre a avaliação psicológica no Brasil. Psicologia: Ciência e Profissão, 30, 192-201. https://doi. org/10.1590/S1414-98932010000500009

Noronha, A. P. P., Rueda, F. J. M., Barros, M. V. C., \& Raad, A. J. (2009). Estudo transversal com estudantes de psicologia sobre conceitos de avaliação psicológica. Psicologia Argumento, 27(56), 77-86. https://doi. org/10.7213/rpa.v27i56.20077

Presidência da República, Casa Civil, Subchefia para Assuntos Jurídicos. (1962). Lei 4.119: Dispõe sobre os cursos de formação em psicologia e regulamenta a profissão de psicólogo. http://www.planalto.gov.br/ ccivil_03/LEIS/1950-1969/L4119.htm

Presidência da República, Casa Civil, Subchefia para Assuntos Jurídicos. (1971). Lei 5.766: Cria o conselho federal e os conselhos regionais de psicologia e dá outras providências. http://www.planalto.gov.br/ccivil_03/LEIS/L5766.htm

Rabelo, I. D. M., Santos, A. M. R., Cattaruzza, M. N., \& Moura, G. C. (2016). Monitoria em psicologia: Uma experiência acadêmica. Cadernos de Graduação, 3(3), 157168.

Rueda, F. J. M., Noronha, A. P. P., Raad, A., \& Varandas, M. (2009). Cuestionario De Competencia En Evaluación Psicológica: Análisis de la estructura interna. Interamerican Journal of Psychology, 43(3), 618-626. http:// pepsic.bvsalud.org/scielo.php?script=sci_abstract\&pid=S0034-96902009000300022\&lng=pt\&nrm=iso

Santos, R. F., Ferreira, A. A., Lima, S. C., Farias, E. S. de, \& Anacleto, G. M. C. (2018). Levantamento de perfil de mulheres universitárias que apresentam medo de dirigir. Revista Científica UMC, edição especial PIBIC, 3(3), 1-4.

Souza, F. M. dos S., \& Barboza, L.C. (2014). A prática de monitoria no ensino de psicologia: Ciência e profissão. Interbio, 8(1), 17-23.

Data de submissão: 08/04/2019 Primeira decisão editorial: 25/06/2019 Aceite em 01/08/2019 